\title{
Martin Weyreuther, Christoph E. Heyde, Michael Westphal: MRI Atlas: Orthopedics And Neurosurgery, The Spine
}

\author{
Hardcover: 296 pages, EUR 123.00, Springer-Verlag, Berlin, Heidelberg, New York, \\ ISBN-10:3540335331, ISBN-13:978-3540335337
}

Pierre Kehr · Denis L. Kaech

Received: 26 October 2008 / Accepted: 27 October 2008 / Published online: 14 November 2008

(C) Springer-Verlag 2008

This is the English version of a textbook written in German and edited in 2006.

Included are chapters about normal anatomy and variants, congenital and developmental anomalies, trauma and fractures, degenerative disorders, inflammatory conditions, tumors and tumor-like lesions and finally about the postoperative spine.

A short definition of the pathoanatomy, the pathomechanism or pathophysiology introduces each topic. Systematically the MR technique, the MR findings and MR pitfalls are listed, with some hints to the additional examinations or sequences that may help to clarify the situation. In two sentences, or on about half a page, the clinical significance is discussed. The following pages of each chapter are dedicated to illustrative cases, with 14 to 154 pictures, depending on the chapter.

After these 7 chapters on 288 pages, 11 book references (from 1989-2002) are recommended for further reading, and depending on the chapter $1-39$ references are proposed, the latest being from 2005. The main key words are finally listed on the last 3 pages.
Imaging being a "visual" speciality, this textbook will be useful for residents in training up to senior general radiologist interested in the possibilities offered my classical MR imaging.

For Spine Surgeons, Neuro- and Orthopedic Surgeons, it is worth looking at and feeding one's visual cortex with some known and some less well known pictures.

Unfortunately, the chapter about postoperative spine with only one reference will not satisfy the eyes of demanding teaching Surgeons, nor help any resident writing papers about this topic. Some illustrations are interesting, e.g., cases of postoperative seromas, but these samples can never compete with a dedicated textbook, e.g., as JR Jinkins's "Posttherapeutic Neurodiagnostic Imaging” published in 1997.

Finally, Spine Surgeons may miss reference about the new upright, multipositional MRI, which allows a noninvasive functional study of the spine-an important diagnostic help prior planning more complex surgery, mainly for the degenerative spine.

Conflict of interest statement No funds were received in support of this study.

P. Kehr $(\bowtie) \cdot$ D. L. Kaech

Strasbourg, France

e-mail: pierre.kehr@argospine.org 